\title{
A novel homozygous premature stop mutation in TNNT2 associates with Feline cardiomyopathy
}

James W McNamara

University of Cincinnati

Maggie Schuckman

MedVet Cincinnati

Richard C Becker

University of Cincinnati

Sakthivel Sadayappan ( $\sim$ sadayasl@ucmail.uc.edu )

University of Cincinnati https://orcid.org/0000-0003-2006-7678

Research article

Keywords: Hypertrophic Cardiomyopathy, Maine Coon, MYBPC3, TNNT2, Sarcomere

Posted Date: January 23rd, 2020

DOI: https://doi.org/10.21203/rs.2.21647/v1

License: (c) (1) This work is licensed under a Creative Commons Attribution 4.0 International License.

Read Full License 
Research Article

A novel homozygous premature stop mutation in TNNT2 associates with Feline cardiomyopathy

James W. McNamara ${ }^{1}$, Maggie Schuckman ${ }^{2}$, Richard C. Becker ${ }^{1}$ and Sakthivel Sadayappan ${ }^{1^{*}}$

${ }^{1}$ Heart, Lung and Vascular Institute, Department of Internal Medicine, Division of Cardiovascular Health and Disease, University of Cincinnati, Cincinnati, OH 45267, USA

${ }^{2}$ MedVet Cincinnati, Department of Cardiology, 3964 Red Bank Rd, Fairfax, OH 45227, USA

*To whom correspondence may be addressed. Email sadayasl@ucmail.uc.edu 


\section{ABSTRACT}

Background: Hypertrophic cardiomyopathy ( $\mathrm{HCM})$ is a genetic disease of the heart and the most common cause of sudden cardiac death in the young. HCM is considered a disease of the sarcomere owing to the large number of mutations in genes encoding sarcomeric proteins. The riddle lies in discovering how these mutations lead to disease. As a result, treatments to prevent and/or treat HCM are limited to invasive surgical myectomies or ablations. Recently, a cohort of Maine Coon cats was identified as carrying an alanine to proline substitution at amino acid 31 of the sarcomeric protein, cardiac myosin binding protein-C, encoded by MYBPC3. Additional mutations in MYBPC3 and MYH7 have also been associated with HCM in cats. In this study, we expand the spectrum of genes associated with HCM in cats.

Results: Next Generation Whole Genome sequencing was performed using DNA isolated from peripheral blood of a Maine Coon with cardiomyopathy that tested negative for the above A31P mutation. Through risk stratification of variants, we identified a novel, homozygous truncating mutation in cardiac troponin-T (TNNT2), also associated with HCM in humans. Both parents tested heterozygous for the mutation, but were unaffected by the disease.

Conclusions: In summary, we are the first to demonstrate the association of TNNT2 mutations and $\mathrm{HCM}$ in a cat, suggesting this gene should be added to the testing panel of genes when performing genetic testing for HCM in cats. 
TNNT2 mutation in feline cardiomyopathy

\section{KEYWORDS}

Hypertrophic Cardiomyopathy; Maine Coon; MYBPC3; TNNT2; Sarcomere 


\section{BACKGROUND}

Hypertrophic cardiomyopathy (HCM) is the most common form of genetic heart disease, affecting at least 1 in 500 humans (1). Defined by an unexplained abnormal thickening of the ventricular walls and possible outflow tract obstruction, no cure is currently available for HCM. $\mathrm{HCM}$ is primarily caused by mutations in genes that encode sarcomeric proteins. In particular, mutations in MYH7 and MYBPC3, encoding beta-cardiac myosin heavy chain and cardiac myosin binding protein-C, are the most commonly mutated genes (2), although other sarcomeric genes, including TNNI3, TNNT2, and MYL2, have also been implicated (3). How mutations in these sarcomeric genes result in the development of HCM remains poorly understood.

In cats, cardiomyopathy is the main cause of cardiovascular disease, with $\mathrm{HCM}$ presenting as the most common form, suggesting that the cat may be an excellent non-rodent animal model of $\mathrm{HCM}$ (4). A variant in MYBPC3, A31P, was previously linked to the development of $\mathrm{HCM}$ in Maine Coon cats (5). This variant occurs within the $\mathrm{C} 0$ domain of cardiac myosin binding protein-C (cMyBP-C), and it is expected to cause disease via structural defects in this domain, which is important for regulation of actomyosin regulation (6). Another variant in MYBPC3, A74T, has also been described across multiple cat breeds. However, indepth follow-up studies demonstrated that this polymorphism is not related to cardiomyopathy $(7,8)$. A third variant in MYBPC3, R820W, has been associated with HCM, most specifically in the Ragdoll (9). More recently, the first variant in MYH7, E1883K, has been associated with HCM in a Domestic Shorthair cat, expanding the spectrum of genes associated with feline cardiomyopathy (10). A full list of mutations associated with feline cardiomyopathy is shown in Table 1.

In this study, a male Maine Coon cat with cardiomyopathy and early congestive heart failure was studied. After initially screening negative for the aforementioned A31P mutation, we performed whole genome sequencing (WGS) to identify potential mutations that may have been causative of this cat's cardiomyopathy. To the best of our knowledge, this is one of the first instances of performing Next Generation Sequencing in the cat (11). As a result, we have identified a novel, autosomal recessive mutation in the sarcomeric TNNT2 gene that appeared to be associated with feline cardiomyopathy. This is the first known record of such mutation in felines, suggesting that TNNT2 should be included as a candidate gene in any future studies. 
TNNT2 mutation in feline cardiomyopathy

\section{RESULTS}

The proband was a male pure-bred Maine Coon cat owned privately. At eight months of age, he presented with atrial and ventricular dilatation and borderline septal hypertrophy, with preserved-to-elevated systolic function (Table 1), when compared to reference echocardiography values for the Maine Coon (12). Diastolic function could not be quantified as a result of $E$ and $A$ wave fusion. Progressive chamber enlargement was noted at 14 months of age, still with borderline septal hypertrophy and preserved systolic function. This was diagnosed as a primary unclassified cardiomyopathy with possible early congestive heart failure.

Furosemide, Benazepril, Spironolactone, and Clopidogrel treatments were then initiated by the veterinarian. This combination appeared to stabilize the progression of the heart disease at 18 months (Table 1).

DNA was isolated from whole peripheral blood from the proband. PCR was performed to produce a 251 amplicon around the A31P variant in MYBPC3 associated with HCM in Maine Coon cats (primer sequences in Table 2, Fig. 2A and B). Sanger sequencing of the PCR product showed no mutation at codon 33 , indicating that the proband did not carry the pathogenic A31P variant in MYBPC3 (Fig. 3D).

As the proband was negative for the A31P mutation in MYBPC3, we questioned whether any additional variants existed that may have been responsible for the cardiomyopathic phenotype in this cat. Accordingly, DNA was freshly prepared for whole genome sequencing (WGS) to identify variants present in the proband. High-quality DNA with an approximate size of 22944bp was sent for library preparation and sequencing by Novogene. Sequences were aligned against the Felis Catus reference genome (Ensemble release 93). The sequencing resulted in 177419470 raw reads, with an average depth of 17.59 reads per base, at an error rate of $0.03 \%$ and $94.19 \%$ of base reads with a Phred score greater than 30 (Fig. $1 \mathrm{~A}-\mathrm{C}$ ). The detected GC content was $43.06 \%$ compared to the reference genome of $41.73 \%$. Together, these data demonstrate high-quality sequencing

Initial analysis reported more than 20,000 genetic variants identified from the proband, compared to the reference genome. We employed a cardiovascular disease risk stratification step in order to enrich for variants associated with cardiovascular disease, searching for the 174 genes currently covered by the Illumina TruSight Cardio Panel (13). This approach reduced the number of variants to 305 (Fig. 2C). Of these 305 potential variants, only one had been annotated as having "high risk" impact. This particular variant revealed a single base pair substitution ( $G$ to $A$ ) within exon 1 at cDNA position 155 of the TNNT2 gene. This substitution resulted in the change of a tryptophan to a stop codon (W52Stop), thus rendering the protein 
product non-functional. It must be noted that the variant in TNNT2 described occurred only in two of the 5 annotated transcripts of the TNNT2 cat gene. Particularly, the N-terminal sequence, containing the variant, is not included in the other three transcripts and encodes a nonconserved protein sequence not found in other species.

Sanger sequencing was performed to confirm the presence of the above W52Stop variant in the proband (primers in Table 2). Strikingly, this sequencing revealed that this variant was, in fact, homozygous, further suggesting the likelihood of its disease association (Fig. 3C). Following this finding, blood samples from both parents of the proband were obtained. Echocardiography did not suggest any cardiac pathology in either of these parents (data not shown). DNA was extracted and PCR performed for Sanger sequencing of the TNNT2 variant. Strikingly, both parents harbored a single copy of the W52Stop variant (Fig. 3A and B). This finding led to investigations into the pedigree of the proband. It was found that the proband was born from consanguineous breeding (Fig. 4). Taken together, these results implicate a novel homozygous truncating mutation in TNNT2 that resulted from interbreeding, as the cause of cardiomyopathy in this Maine Coon cat.

\section{DISCUSSION}

In this study, we identified a novel homozygous mutation in TNNT2 associated with a case of feline cardiomyopathy that progressed to an early heart failure phenotype. To the best of our knowledge, this is the first report of a thin filament mutation associated with feline cardiac disease. Other mutations associated with feline cardiomyopathy have previously been described in MYBPC3 and MYH7 $(5,10)$. Importantly, mutations in TNNT2 have been strongly implicated in the development of HCM and dilated cardiomyopathy (DCM) $(14,15)$. In humans, these TNNT2 mutations have been described as phenotypically mild compared to $M Y H 7$ mutations, but with a higher rate of sudden cardiac death (15). Furthermore, a truncating mutation in TNNT2, resulting from an intronic splice site mutation has also been described (16). Taken together, these data support our finding that the TNNT2 truncating mutation we describe is the most likely cause of this case of feline cardiomyopathy.

The TNNT2 gene encodes the cardiac-specific sarcomeric protein, cardiac troponin- $T$ (cTnT) (17). Cardiac troponin-C and -I (cTnC and cTnl, respectively), together with cTnT, make up the troponin complex of the heart (18). The troponin complex associates with the thin filaments, and cTnT is thought to play an important role in anchoring the complex to both actin and tropomyosin (18). When calcium is released from the sarcoplasmic reticulum, it binds to and induces a structural change in cTnC (19), ultimately leading to the movement of 
tropomyosin, allowing the formation of cross-bridges (20). cTnT has been demonstrated to regulate the calcium sensitivity of actomyosin ATPase and force (21). Thus, cTnT has a central role in regulation contraction and relaxation of the heart.

Homozygous knockout of the TNNT2 gene in mice is embryonically lethal $(22,23)$. As such, it is unlikely that the two isoforms of TNNT2 that are affected by the truncating mutation comprise the entire pool of heart cTnT. Rather, it is possible that these two isoforms of cTnT represent a portion of the total cTnT and that loss of these isoforms results in haploinsufficiency of cTnT. This small change in cTnT levels would result in an abnormal stoichiometry of thin filament proteins in the sarcomere, which may be sufficient to cause cardiomyopathy. Indeed, haploinsufficiency of cTnT has previously been associated with cardiomyopathy (24).

Furthermore, the incorporation of less than $5 \%$ of C-terminally truncated cTnT was sufficient to cause cardiomyopathy in mice (25). Since the proband's symptoms are currently managed via treatment, we are unable to confirm this. Unfortunately, we did not have access to DNA from the proband's littermate, but we predict it would be either negative or heterozygous for the W52Stop mutation, as we understand it is currently healthy. Alternatively, two single nucleotide variants were also identified in MYBPC3 that were classified as having moderate risk. These variants were a proline substituted to a leucine at position 922 (P922L) and an alanine for a threonine at amino acid 1037 (A1037T), occurring within domains C7 and C8 of cMyBP-C, respectively. However, further investigation of these variants revealed that these amino acids are not evolutionarily conserved, and, furthermore, the substituted amino acids matched those of either human or mouse, effectively ruling these $M Y B P C 3$ variants out as disease related.

\section{CONCLUSIONS}

We have identified a novel, homozygous mutation in the sarcomeric gene TNNT2, which is associated with cardiomyopathy in a Maine Coon cat. The homozygosity of this mutation resulted from inbreeding. This study is the first to describe a mutation in TNNT2 that associates with feline cardiomyopathy, suggesting that this gene should be included in genetic testing in felines. Additionally, breeders should be strongly reminded of the dangers associated with close generational consanguineous breeding. 


\section{METHODS}

\section{Phenotyping, Blood Collection and DNA extraction}

All procedures followed the protocol approved by the Institutional Animal Care and Use Committee of the University of Cincinnati and complied with the Guide for the Use and Care of Laboratory Animals published by the National Institutes of Health. The proband's owner contacted us to determine if there was a genetic association of HCM. To establish a full research study, a Material Transfer Agreement was initiated between the proband's owner and the University of Cincinnati. Furthermore, researchers had no interaction with the cats, just receiving the blood samples. All interaction with animals were performed by board certified veterinarians and therefore, there was no animal protocol included in the present study. As part of the diagnosis and treatment for the proband, regular checkups with echocardiography were performed by a boarded veterinary cardiologist using standard echocardiographic techniques and references from a population of normal Maine Coon cats $(12,26)$. Peripheral blood was collected by a certified veterinarian or veterinarian nurse at each animal's local hospital following routine check-up. Blood collected locally was transported back to the laboratory on wet ice. Blood that was collected at further distances was shipped on cold blocks by overnight shipment. Upon receipt at the laboratory, all blood samples were aliquoted into $200 \mu$ l samples and stored at $-80^{\circ} \mathrm{C}$ until use. DNA was extracted from a single tube using the Qiagen QIAamp. DNA Mini and Blood DNA isolation kit, following the manufacturer's protocol. Concentration and purity of DNA was analyzed by Nanodrop. For the sample sent for whole genome sequencing, DNA integrity and concentration were also determined using the Agilent 2100 Bioanalyzer and Qubit fluorometer, respectively.

\section{Whole Genome Sequencing and Analysis}

DNA extracted from the whole blood of the proband was submitted to Novogene Co. Ltd. for whole genome sequencing (WGS). DNA quality and quantity were confirmed by Agarose gel electrophoresis and Qubit fluorometer. A total amount of $1.5 \mu \mathrm{g}$ high quality genomic DNA was randomly sheared in to short fragments of approximately $350 \mathrm{bp}$ and used for library construction using the NEBNext DNA Library Prep Kit. Briefly, following end repairing, dA-tailing, and ligation with NEBNext adapter, the fragments were PCR-enriched by P5 and indexed P7 oligos. The concentration of the DNA library was quantified using the Qubit 2.0 fluorometer and diluted to $1 \mathrm{ng} / \mu \mathrm{l}$. Following dilution, the insert size of the library was assessed with the Agilent 2100 bioanalyzer, and quantitative real-time PCR (qPCR) was performed to detect the effective concentration of the prepared library. Following enrichment and indexing, pair-end sequencing 
of the qualified library was performed on an Illumina HiSeq X Ten with a read length of PE150bp at each end.

\section{Bioinformatics analysis}

The original sequencing data acquired by high-throughput sequencing platforms (e.g., Illumina $\mathrm{HiSeq}^{\mathrm{TM}} / \mathrm{NovaSeq}^{\mathrm{TM}}$ ) recorded in image files are first transformed to sequence reads by base calling with the CASAVA software. Information on sequences and corresponding sequencing quality is stored in a FASTQ file. Following quality control of raw sequencing data for clean data filtration, each clean read was mapped to the reference genome (Felis_Catus_9.0, Ensembl release 93) using the BWA software (27), and the mapping rate and coverage were counted according to the alignment results. Duplicates were removed by SAMTOOLS (28). Single nucleotide polymorphism (SNP) and InDel variants were detected using the GATK software (29). These SNPs were annotated using ANNOVAR as previously described (30).

\section{Sanger Sequencing}

To test, or confirm, the presence of a genetic variant in parents of the proband, PCR was performed using a high-fidelity Taq polymerase to specifically amplify sequence around the variant. The PCR product was purified and sent for Sanger sequencing using Cincinnati Children's Hospital DNA Sequencing and Genotyping Core Facility.

\section{ABBREVIATIONS}

HCM, Hypertrophic cardiomyopathy

MYH7, Gene encoding $\beta$-myosin heavy chain

MYBPC3, Gene encoding cardiac myosin binding protein-C

TNNT2, Gene encoding cardiac troponin-T

cMyBP-C, Cardiac myosin binding protein-C

cTnT, Cardiac troponin-T

cTnl, Cardiac troponin-I

cTnC, Cardiac troponin-C

WGS, Whole genome sequencing

SNP, Single nucleotide polymorphism 


\section{DECLARATIONS}

\section{Ethics Approval and Consent to Participate}

This study was approved by the University of Cincinnati by the Institutional Animal Care and Use Committee of the University of Cincinnati and complied with the Guide for the Use and Care of Laboratory Animals published by the National Institutes of Health. Informed consent (material transfer agreement) was established between the owners and the University of Cincinnati to collect samples and data of the animals that were used in this study.

\section{Consent for Publication}

Not applicable.

\section{Availability of Data and Materials}

The datasets used and/or analyzed during the current study are available from the corresponding author on reasonable request.

\section{Competing Interests}

Dr. Sadayappan provided consulting and collaborative research studies to the Leducq Foundation, Red Saree Inc., Greater Cincinnati Tamil Sangam, MyoKardia, Merck and Amgen, but such work is unrelated to the content of this manuscript. No other disclosures are reported.

\section{Funding}

Whole genome sequencing of the proband was funded by a donation from Kathleen and Michael Janson, parents of the proband. Dr. McNamara salary was supported by an American Heart Association Postdoctoral Fellowship (17POST33630095). Dr. Sadayappan percentage of efforts, in terms of his salary, was received from the following grants: National Institutes of Health grants R01 HL130356, R56 HL139680, R01 AR067279, R01 HL105826 and R01 HL143490; and transformation (19TPA34830084) awards; and MyoKardia, AstraZeneca, Merck and Amgen.

Author Contributions: JM and SS designed research. JM and MS performed research. JM, MS, and SS analyzed data. JM and SS wrote the manuscript. MS and RB edited the manuscript. RB provided project oversight for clinical data. All authors read and approved the final manuscript. 


\section{Acknowledgements}

The authors would like to thank the staff at Warm Animal, Cincinnati; Big Creek, Cleveland; and Friendship, Washington DC veterinary hospitals for performing the blood draws. We also thank Linda Komar and Joseph Keyerleber for organizing the shipments of the proband's parents blood. Sanger sequencing was performed by Cincinnati Children's Hospital DNA sequencing and genotyping core. 


\section{REFERENCES}

1. Ho CY. New Paradigms in Hypertrophic Cardiomyopathy: Insights from Genetics. Progress in pediatric cardiology. 2011;31(2):93-8.

2. Viswanathan SK, Sanders HK, McNamara JW, Jagadeesan A, Jahangir A, Tajik AJ, et al. Hypertrophic cardiomyopathy clinical phenotype is independent of gene mutation and mutation dosage. PloS one. 2017;12(11):e0187948.

3. Brouwer WP, van Dijk SJ, Stienen GJ, van Rossum AC, van der Velden J, Germans T. The development of familial hypertrophic cardiomyopathy: from mutation to bedside. European journal of clinical investigation. 2011;41(5):568-78.

4. Ferasin L, Sturgess CP, Cannon MJ, Caney SM, Gruffydd-Jones TJ, Wotton PR. Feline idiopathic cardiomyopathy: a retrospective study of 106 cats (1994-2001). Journal of feline medicine and surgery. 2003;5(3):151-9.

5. Meurs KM, Sanchez X, David RM, Bowles NE, Towbin JA, Reiser PJ, et al. A cardiac myosin binding protein $C$ mutation in the Maine Coon cat with familial hypertrophic cardiomyopathy. Human molecular genetics. 2005;14(23):3587-93.

6. van Dijk SJ, Bezold Kooiker K, Mazzalupo S, Yang Y, Kostyukova AS, Mustacich DJ, et al. The A31P missense mutation in cardiac myosin binding protein $\mathrm{C}$ alters protein structure but does not cause haploinsufficiency. Archives of biochemistry and biophysics. 2016;601:133-40.

7. Longeri M, Ferrari $P$, Knafelz P, Mezzelani A, Marabotti A, Milanesi L, et al. Myosinbinding protein C DNA variants in domestic cats (A31P, A74T, R820W) and their association with hypertrophic cardiomyopathy. Journal of veterinary internal medicine. 2013;27(2):275-85.

8. Wess G, Schinner C, Weber K, Kuchenhoff H, Hartmann K. Association of A31P and A74T polymorphisms in the myosin binding protein $\mathrm{C} 3$ gene and hypertrophic cardiomyopathy in Maine Coon and other breed cats. Journal of veterinary internal medicine. 2010;24(3):527-32.

9. Meurs KM, Norgard MM, Ederer MM, Hendrix KP, Kittleson MD. A substitution mutation in the myosin binding protein $\mathrm{C}$ gene in ragdoll hypertrophic cardiomyopathy. Genomics. 2007;90(2):261-4.

10. Schipper T, Van Poucke M, Sonck L, Smets P, Ducatelle R, Broeckx BJG, et al. A feline orthologue of the human MYH7 c.5647G>A (p.(Glu1883Lys)) variant causes hypertrophic cardiomyopathy in a Domestic Shorthair cat. European journal of human genetics. 2019;27(11):1724-1730.

11. Ontiveros ES, Ueda Y, Harris SP, Stern JA. Precision medicine validation: identifying the MYBPC3 A31P variant with whole-genome sequencing in two Maine Coon cats with hypertrophic cardiomyopathy. Journal of feline medicine and surgery. 2018:1098612x18816460. 
12. Drourr L, Lefbom BK, Rosenthal SL, Tyrrell WD, Jr. Measurement of M-mode echocardiographic parameters in healthy adult Maine Coon cats. Journal of the American Veterinary Medical Association. 2005;226(5):734-7.

13. Pua CJ, Bhalshankar J, Miao K, Walsh R, John S, Lim SQ, et al. Development of a Comprehensive Sequencing Assay for Inherited Cardiac Condition Genes. Journal of cardiovascular translational research. 2016;9(1):3-11.

14. Pasquale F, Syrris P, Kaski JP, Mogensen J, McKenna WJ, Elliott P. Long-term outcomes in hypertrophic cardiomyopathy caused by mutations in the cardiac troponin $T$ gene. Circulation Cardiovascular genetics. 2012;5(1):10-7.

15. Watkins H, McKenna WJ, Thierfelder L, Suk HJ, Anan R, O'Donoghue A, et al. Mutations in the genes for cardiac troponin $\mathrm{T}$ and alpha-tropomyosin in hypertrophic cardiomyopathy. The New England journal of medicine. 1995;332(16):1058-64.

16. Thierfelder L, Watkins H, MacRae C, Lamas R, McKenna W, Vosberg HP, et al. Alphatropomyosin and cardiac troponin $\mathrm{T}$ mutations cause familial hypertrophic cardiomyopathy: a disease of the sarcomere. Cell. 1994;77(5):701-12.

17. Wei B, Jin JP. TNNT1, TNNT2, and TNNT3: Isoform genes, regulation, and structurefunction relationships. Gene. 2016;582(1):1-13.

18. Gomes AV, Potter JD, Szczesna-Cordary D. The role of troponins in muscle contraction. IUBMB life. 2002;54(6):323-33.

19. Vinogradova MV, Stone DB, Malanina GG, Karatzaferi C, Cooke R, Mendelson RA, et al. $\mathrm{Ca}(2+)$-regulated structural changes in troponin. Proceedings of the National Academy of Sciences of the United States of America. 2005;102(14):5038-43.

20. Boussouf SE, Maytum R, Jaquet K, Geeves MA. Role of tropomyosin isoforms in the calcium sensitivity of striated muscle thin filaments. Journal of muscle research and cell motility. 2007;28(1):49-58.

21. Gomes AV, Liang J, Potter JD. Mutations in human cardiac troponin I that are associated with restrictive cardiomyopathy affect basal ATPase activity and the calcium sensitivity of force development. The Journal of biological chemistry.

2005;280(35):30909-15.

22. Ahmad F, Banerjee SK, Lage ML, Huang XN, Smith SH, Saba S, et al. The role of cardiac troponin T quantity and function in cardiac development and dilated cardiomyopathy. PloS one. 2008;3(7):e2642.

23. Nishii K, Morimoto S, Minakami R, Miyano Y, Hashizume K, Ohta M, et al. Targeted disruption of the cardiac troponin $T$ gene causes sarcomere disassembly and defects in heartbeat within the early mouse embryo. Developmental biology. 2008;322(1):65-73.

24. Bollen IAE, Schuldt M, Harakalova M, Vink A, Asselbergs FW, Pinto JR, et al. Genotypespecific pathogenic effects in human dilated cardiomyopathy. J Physiol.

2017;595(14):4677-93. 
25. Tardiff JC, Factor SM, Tompkins BD, Hewett TE, Palmer BM, Moore RL, et al. A truncated cardiac troponin T molecule in transgenic mice suggests multiple cellular mechanisms for familial hypertrophic cardiomyopathy. The Journal of clinical investigation. 1998;101(12):2800-11.

26. Thomas WP, Gaber CE, Jacobs GJ, Kaplan PM, Lombard CW, Moise NS, et al. Recommendations for standards in transthoracic two-dimensional echocardiography in the dog and cat. Echocardiography Committee of the Specialty of Cardiology, American College of Veterinary Internal Medicine. Journal of veterinary internal medicine. 1993;7(4):247-52.

27. Li H, Durbin R. Fast and accurate short read alignment with Burrows-Wheeler transform. Bioinformatics (Oxford, England). 2009;25(14):1754-60.

28. Li H, Handsaker B, Wysoker A, Fennell T, Ruan J, Homer N, et al. The Sequence Alignment/Map format and SAMtools. Bioinformatics (Oxford, England). 2009;25(16):2078-9.

29. DePristo MA, Banks E, Poplin R, Garimella KV, Maguire JR, Hartl C, et al. A framework for variation discovery and genotyping using next-generation DNA sequencing data. Nature genetics. 2011;43(5):491-8.

30. Wang K, Li M, Hakonarson H. ANNOVAR: functional annotation of genetic variants from high-throughput sequencing data. Nucleic acids research. 2010;38(16):e164. 
TNNT2 mutation in feline cardiomyopathy

\section{TABLES}

Table 1. The current list of known mutations that associate with feline cardiomyopathy.

\begin{tabular}{llll}
\hline Gene & Mutation & Breed & Reference \\
\hline \hline MYBPC3 & A31P & Maine Coon & {$[6]$} \\
MYBPC3 & R820W & Ragdoll & {$[10]$} \\
MYH7 & E1883K & Domestic Shorthair & {$[11]$} \\
\hline
\end{tabular}


Table 2. Echocardiography parameters from serial measurements performed by a board certified veterinary cardiologist. Significant dilatation of all cardiac chambers was noted with normal to elevated systolic function. Treatments administered are listed.

\begin{tabular}{|c|c|c|c|c|c|}
\hline \multirow{2}{*}{$\begin{array}{l}\text { Parameter } \\
\text { Age (month) }\end{array}$} & \multicolumn{4}{|c|}{ Proband } & \multirow{2}{*}{$\begin{array}{l}\text { Reference } \\
>12\end{array}$} \\
\hline & 10 & 14 & 18 & 24 & \\
\hline IVSd (mm) & 6 & 5.9 & 5.88 & 5.94 & $3.9-4.1$ \\
\hline LVIDd (mm) & 19.3 & 19.9 & 21.8 & 21.7 & $18.1-18.9$ \\
\hline $\begin{array}{l}\text { LVPWd } \\
(\mathrm{mm})\end{array}$ & 5.1 & 5.5 & 5.27 & 5.55 & $4.2-4.4$ \\
\hline IVSs (mm) & 9.6 & 9.2 & $\mathrm{~N} / \mathrm{A}$ & $\mathrm{N} / \mathrm{A}$ & $7.2-7.8$ \\
\hline LVIDs (mm) & 9 & 9.6 & 7.79 & 8.11 & $8.5-9.3$ \\
\hline $\begin{array}{l}\text { LVPWs } \\
(\mathrm{mm})\end{array}$ & 9.6 & 9.4 & $\mathrm{~N} / \mathrm{A}$ & $\mathrm{N} / \mathrm{A}$ & $7.8-8.2$ \\
\hline$\% F S$ & 60.6 & 59.5 & 64.2 & 62.6 & $\begin{array}{l}50.35- \\
53.35\end{array}$ \\
\hline $\mathrm{LA}(\mathrm{mm})$ & 17.6 & 19.8 & 18.9 & 19.7 & $13.4-14.0$ \\
\hline LA/Ao & 1.33 & 1.58 & 1.53 & 1.44 & $1.20-1.26$ \\
\hline Treatments & & $\begin{array}{l}\text { Furosemide, } \\
\text { Benazepril, } \\
\text { Clopidogrel }\end{array}$ & $\begin{array}{l}\text { Spironolactone, } \\
\text { Furosemide, } \\
\text { Benazepril, } \\
\text { Clopidogrel }\end{array}$ & $\begin{array}{l}\text { Spironolactone, } \\
\text { Furosemide, } \\
\text { Benazepril, } \\
\text { Clopidogrel }\end{array}$ & \\
\hline \multicolumn{6}{|c|}{$\begin{array}{l}\text { IVS, interventricular septal thickness; LVID, left ventricular internal diameter; LVPW, left } \\
\text { ventricular posterior wall thickness; FS, fractional shortening; LA, left atrial dimension; LA/Ao } \\
\text { Left atrium-to-aortic root ratio; d, diastole; s, systole. N/A represents data not available. }\end{array}$} \\
\hline
\end{tabular}


Table 3. The list of primer sequences used in this study to test the presence of variants in MYBPC3 and TNNT2.

\begin{tabular}{|l|l|l|}
\hline Primer Name & Primer Sequence & \multirow{2}{*}{ Amplicon size (bp) } \\
\hline \hline Cat_A31P_F & AGTCTCAGCCTTCAGCAAGAAGCC & \multirow{2}{*}{$252 \mathrm{bp}$} \\
\cline { 1 - 2 } Cat_A31P_R & GGTCAAACTTGACCTTGGAGGAGCC & \multirow{2}{*}{$287 \mathrm{bp}$} \\
\cline { 1 - 2 } Cat_TNNT2_F & TGAGTGGATGTGGCTGTGTT & \\
\cline { 1 - 2 } Cat_TNNT2_F & CTCTTCTGCTGCTGCCTCTT & \\
\hline
\end{tabular}




\section{FIGURE LEGENDS}

FIGURE 1. Quality control of Next Generation Sequencing results. A, From 177419470 sequencing reads, $99.95 \%$ were clean reads, while just $0.05 \%$ contained adapter-related contaminations. B, Quantification of sequencing quality distribution along reads demonstrated high-quality scores along entire reads with $94.19 \%$ of base reads having a Phred score greater than 30 . C, Error rate distribution along reads demonstrated high-fidelity reads with an overall error rate of $0.03 \%$.

FIGURE 2. Representative echocardiography and outline of cardiovascular disease risk stratification of genetic variants. Representative parasternal long axis B-Mode (A) and M-Mode (B) images of proband. C, Following isolation of DNA from blood of proband, DNA QC was performed in both institutional and company laboratories. Library preparation and sequencing was performed, which identified more than 20,000 genetic variants. Gene stratification was performed based on the presence of genes in the 174 cardiopanel gene list. This reduced the list to 304 variants. Of these, only one variant had been classified as "high risk", namely the W52Stop mutant in TNNT2.

FIGURE 3. Sanger sequencing to test presence of the genetic variants. Sequencing of the proband's sire $(\mathbf{A})$ and dam $(\mathbf{B})$ revealed that both were heterozygous for the W52Stop variant. C, Sanger sequencing of the proband showed homozygosity for the W52Stop. D, Chromatogram of MYBPC3 A31P demonstrated that the proband was negative for this variant.

FIGURE 4. Family pedigree of affected proband. Circles and squares represent males and females, respectively, while blue and red represent unaffected and affected individuals, respectively. A male Maine Coon, heterozygous for the W52Stop variant, was mated to a female Maine Coon, which resulted in one female offspring. This female offspring, also heterozygous, was then bred with the original male, producing two offspring. The male offspring was the cardiomyopathic proband in this study and was homozygous for the W52Stop variant. * indicates that samples from these cats were not available at the time of the study. 


\section{Figure 1}
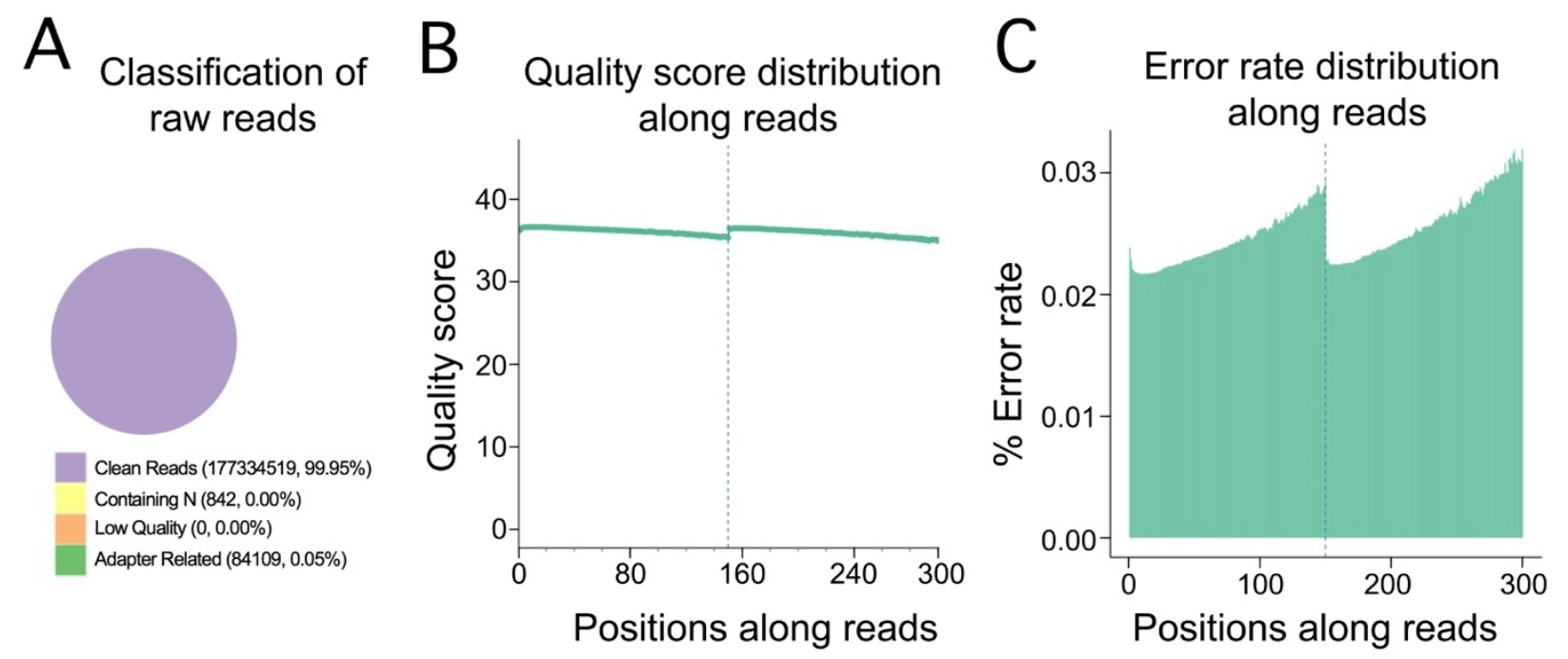
Figure 2
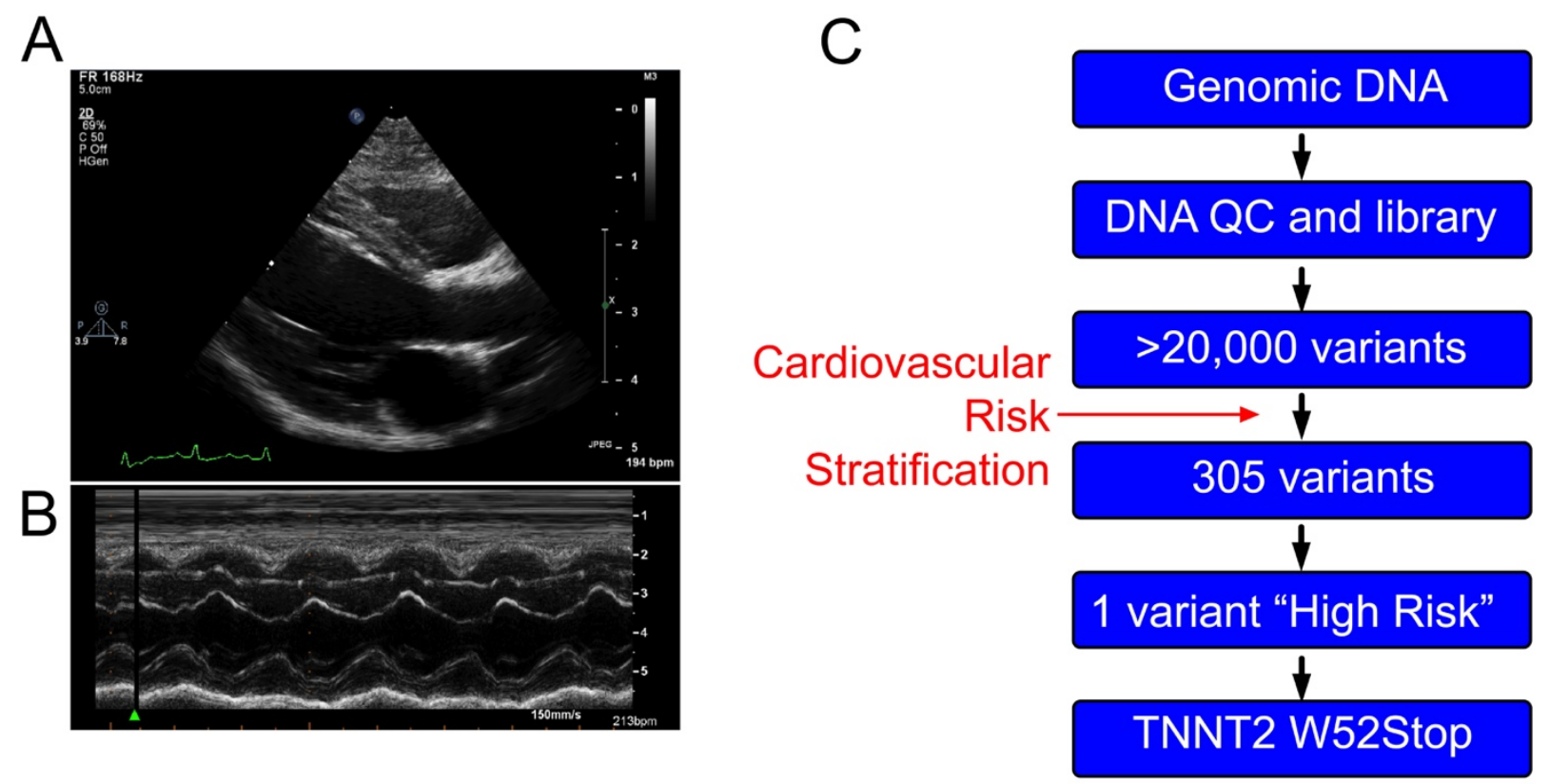
Figure 3

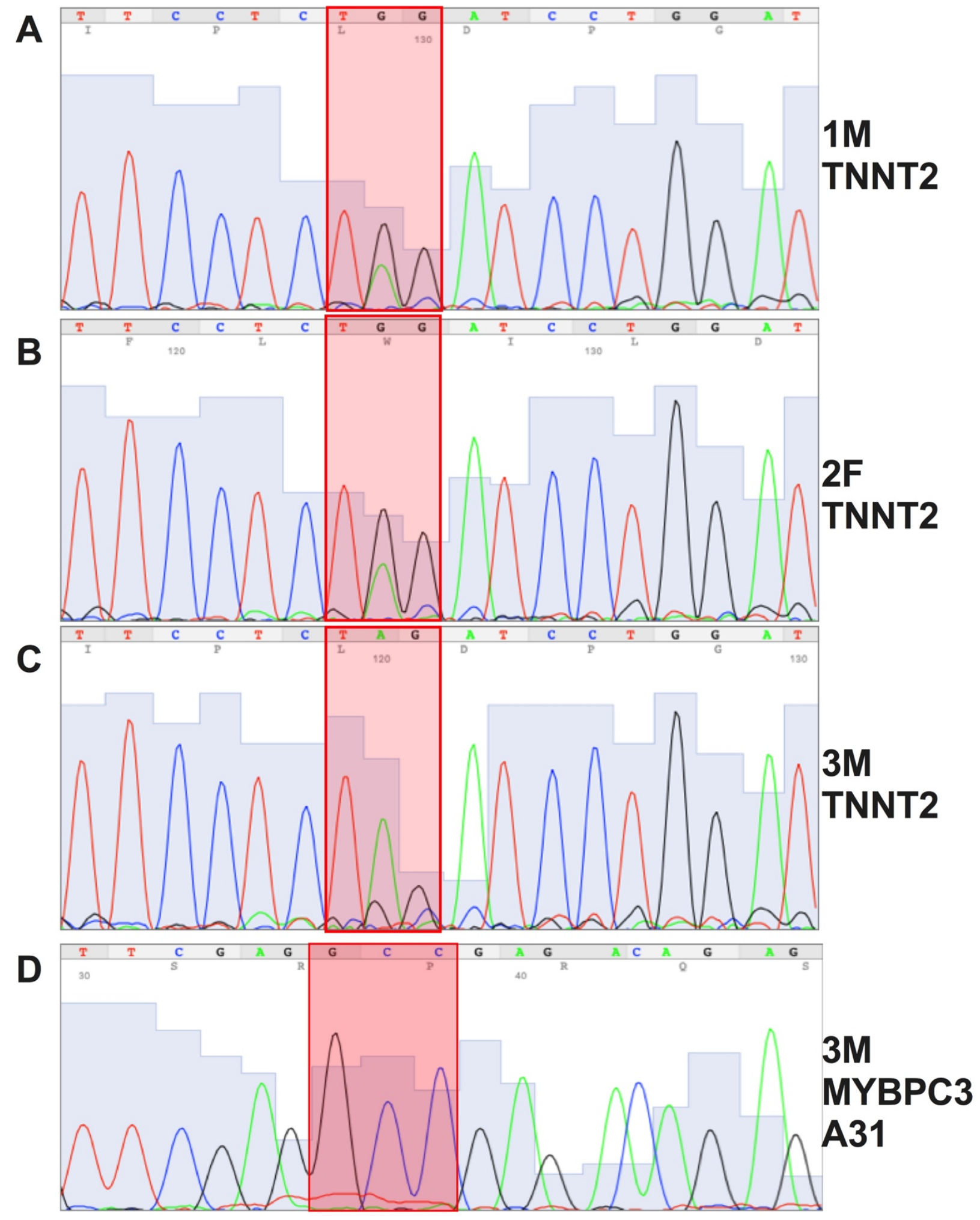


Figure 4

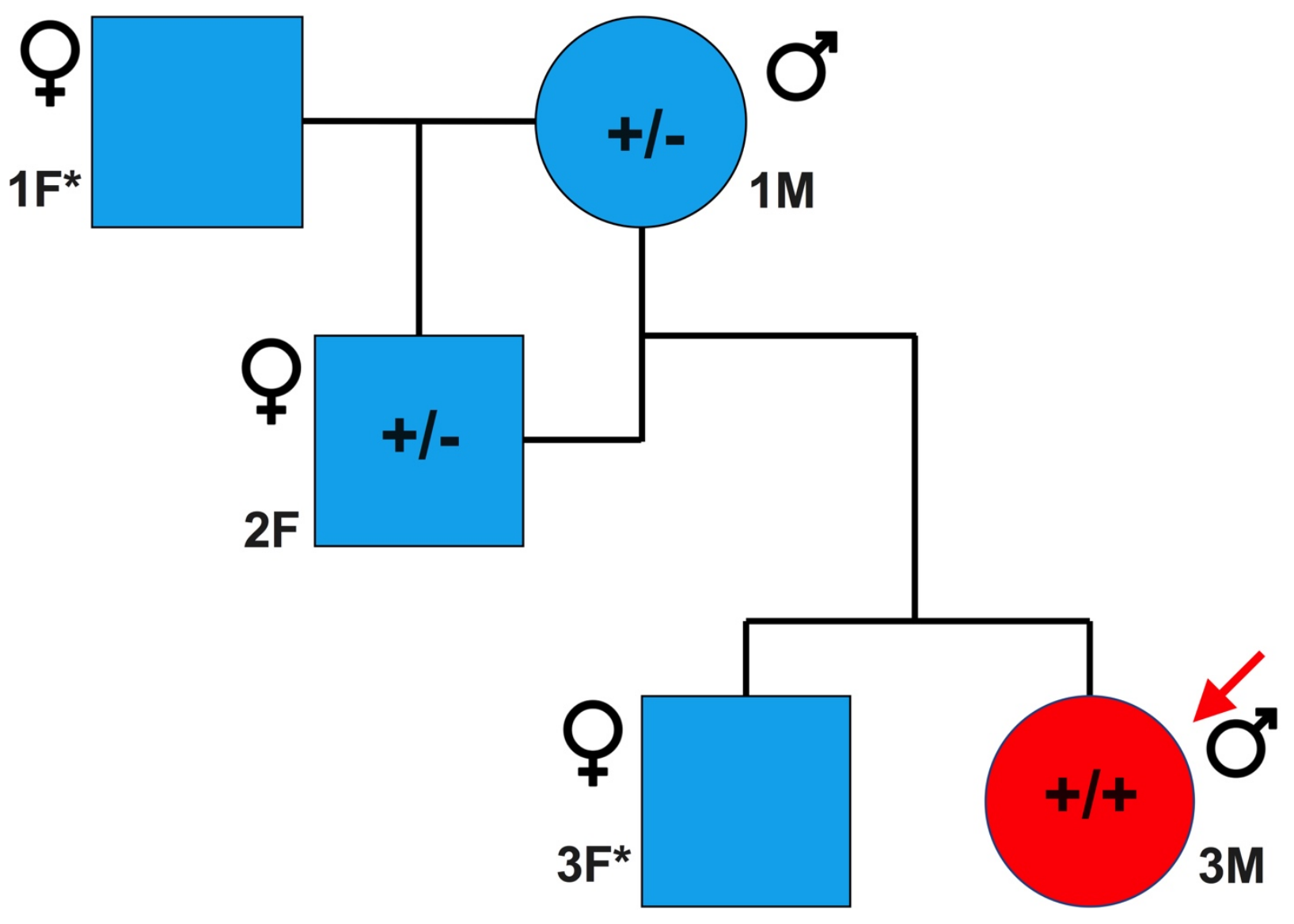

